\title{
]jfis
}

\section{Fuzzy Parameterized Bipolar Fuzzy Soft Expert Set and Its Application in Decision Making}

\author{
Muhammad Ibrar ${ }^{1}$, Asghar Khan ${ }^{1}$, Sajjad Khan ${ }^{2}$, and Fatima Abbas ${ }^{3}$ \\ ${ }^{1}$ Department of Mathematics, Abdul Wali Khan University, Mardan, Pakistan \\ ${ }^{2}$ Department of Mathematics, Hazara University, Hazara, Pakistan \\ ${ }^{3}$ Department of Mathematics, Gomal University, Dera Ismail Khan, Pakistan
}

Received: Apr. 28, 2019

\begin{abstract}
In this paper, we present the study of fuzzy parameterized bipolar fuzzy soft expert sets and define some of its basic operations. We validated the basic properties and essential laws related to this methodology. Lastly, we presented some possible applications in decision-making problems using a generalized algorithm.
\end{abstract}

Keywords: Bipolar fuzzy soft sets, Bipolar fuzzy soft expert sets, Fuzzy parameterized bipolar fuzzy soft expert sets.

\section{Introduction}

All existing theories on uncertainty have their own flaws as pointed out by Molodtsov [1]. Molodtsov [1] was the pioneer who introduced the idea of soft sets. In [2], the authors extended the work of Molodtsov [1] and established the theory of fuzzy soft sets. Meanwhile, Alkhazaleh et al. [3] established the notion of fuzzy parameterized interval-valued fuzzy soft set and discussed its application in a decision-making problem. Cagman and his colleagues [4, 5] introduced the concept of fuzzy parameterized soft sets and fuzzy parameterized fuzzy soft sets and discussed the associated properties. Bashir and Salleh [6] pioneered the concept of fuzzy parameterized soft expert sets. Hazaymeh et al. [7] then extended the work of Bashir and Salleh [6] and discussed the theory of fuzzy parameterized fuzzy soft expert sets. The idea of fuzzy parameterized intuitionistic fuzzy soft expert sets and its application in decision making was first explored by Selvachandranwe and Salleh [8]. The idea of bipolar valued fuzzy sets was first established by Lee [9].

In this paper, we extend the concept of fuzzy parameterized fuzzy soft expert set to the bipolar-valued fuzzy set introduced by Lee [10], and we introduce the fuzzy parameterized cCThis is an Open Access article distributed under the terms of the Creative Commons Attribution Non-Commercial License (http://creativecommons.org/licenses/ by-nc/3.0// which permits unrestricted noncommercial use, distribution, and reproduction in any medium, provided the original work is properly cited. bipolar fuzzy soft expert set (FPBFSES). We investigated the basic properties of this set such as De Morgan's law and applied it to a decision-making problem, i.e., the home buying process.

\section{Methods}

Many researchers have studied the notion of soft expert sets and fuzzy soft expert sets. There 
continues to be growing research interest in this field. In this paper, we extend the work of Hazaymeh et al. [7] and introduce the notion of FPBFSES.

The paper is divided into different sections to discuss the various topics. In Section 2, the basic concepts of soft sets, bipolar fuzzy sets, bipolar fuzzy soft sets, soft expert sets, fuzzy parameterized fuzzy soft sets, and fuzzy parameterized fuzzy soft expert sets are provided. The definition and basic concepts of FPBFSES are discussed in Section 3. In Section 4, the fundamental operations on FPBFSES such as the union, intersection, complement, AND operator, OR operator and its properties are discussed. In Section 5, we design a generalized algorithm in FPBFSES to solve a decision-making problem, i.e., the home buying process. In the last section, we present the conclusion of the study.

\section{Results and Discussions}

Basic information on soft sets, bipolar fuzzy sets, bipolar fuzzy soft sets, soft expert sets, fuzzy parameterized fuzzy soft sets, and fuzzy parameterized fuzzy soft expert sets is provided.

Definition 3.1 [1] . Let $\mathrm{F}$ be the mapping given by : $U \longrightarrow$ $P(U)$ where $P(U)$ is a power set of $U$. The pair $(U, F)$ is called a soft set over $U$.

Definition 3.2 [9]. A bipolar-valued fuzzy set A defined over $U$ is a mapping of the form

$$
\begin{aligned}
& A=\left\{\left(x, f^{+}{ }_{A}(x), f^{-}{ }_{A}(x)\right): x \in U\right\}, \\
& f_{A}^{+}: U \longrightarrow[0,1], \\
& f_{A}^{-}: U \longrightarrow[-1,0],
\end{aligned}
$$

where $f_{A}^{+}(x)$ represents the degree that $x$ satisfies a property, while $f_{A}^{-}(x)$ represents the degree that $\mathrm{x}$ satisfies an implicit counter-property.

With $A$ and $B$ as bipolar fuzzy sets defined over the universal set $U$, they can be expressed as follows:

$$
\begin{aligned}
& A=\left\{\left(a, f_{A}^{+}(a), f_{A}^{-}(a)\right): a \in U\right\}, \\
& B=\left\{a, g_{B}^{+}(a), g_{B}^{-}(a): a \in U\right\} .
\end{aligned}
$$

A bipolar fuzzy set $A$ is a subset of a bipolar fuzzy set $B$ if $f_{A}^{+}(a) \leq g_{B}^{+}(a)$ and $f_{A}^{-}(a) \geq g_{B}^{-}(a) \forall a \in U$. They are equal if $A$ is subset of $B$ and $B$ is subset of $A$.

Definition 3.3 [9]. The complement, union, and intersection of two bipolar fuzzy sets $A$ and $B$ are defined as follows:

$$
\begin{aligned}
& A^{c}=\left\{x, f_{A^{c}}^{+}(x), f_{A^{c}}^{-}(x): x \in U\right\}, \\
& f_{A^{c}}^{+}(x)=1-f_{A}^{+}(x), f_{A^{c}}^{-}(x)=-1-f_{A}^{-}(x), \\
& A \cup B=\left\{x,\left(f_{A}^{+}(x) \vee g_{B}^{+}(x)\right),\left(f_{A}^{-}(x) \wedge g_{B}^{-}(x)\right): x \in U\right\}, \\
& A \cap B=\left\{x,\left(f_{A}^{+}(x) \wedge g_{B}^{+}(x)\right),\left(f_{A}^{-}(x) \vee g_{B}^{-}(x)\right): x \in U\right\} .
\end{aligned}
$$

Definition $3.4[10]$. Consider a set of universe $U$ and a set of parameter $E$. Suppose $P(U)$ represents the set of all bipolar fuzzy sets on $U$. Let $\mathrm{F}$ be a function given by $F: A \longrightarrow P(U)$. Then, the pair $(F, A)$ is called a bipolar fuzzy soft set (BFSS) over $\mathrm{U}$ where $A \subseteq E$.

Definition 3.5 [10]. A BFSS $(F, A)$ is called a null BFSS if $F(e)=\emptyset$ for all $e \in A$.

Definition 3.6 [10]. A BFSS $(F, A)$ is called an absolute BFSS if $F(e)=B^{U} \forall e \in A$, where $B^{U}$ is the collection of all bipolar fuzzy subset of $U$.

Definition 3.7 [10]. Let $(G, X)$ and $(H, Y)$ be two BFSSs of $U$. Then, the union of $(G, X)$ and $(H, Y)$, represented by $(G, X) \bigcup(H, Y)$ is a BFSS classified as $(G, X) \bigcup(H, Y)=$ $(I, Z)$, where $Z=X \cup Y$ and $t \in Z$,

$$
I(a)= \begin{cases}G(t), & \text { if } t \in X-Y, \\ H(t), & \text { if } t \in Y-X \\ G(t) \cup H(t), \text { if } t \in X \cap Y . & \end{cases}
$$

Definition 3.8 [10]. Let $(G, X)$ and $(H, Y)$ be two BFSSs of $\mathrm{U}$. Then, the intersection of $(G, X)$ and $(H, Y)$, represented by $(G, X) \bigcap(H, Y)$ is a BFSS classified as $(G, X) \bigcap(H, Y)=$ $(I, Z)$ where $Z=X \cup Y$ and $t \in Z$,

$$
I(a)=\left\{\begin{array}{ll}
G(t), & \text { if } t \in X-Y, \\
H(t), & \text { if } t \in Y-X, \\
G(t) \cap H(t), & \text { if } t \in X \cap Y .
\end{array} .\right.
$$

Definition $3.9[11]$. Let $F$ be a function given by $F: A \longrightarrow$ $P(U)$. Then, the pair $(F, A)$ is said to be a soft expert set over $U$, where $A \subseteq Z=E \times X \times O$.

Definition $3.10[12]$. Consider a set of universe $U$, a set of parameter $E$, a set of experts $X$ and a set of options $O$. Let $Z=E \times X \times O$ and $A \subseteq Z$. Suppose $F: A \longrightarrow B^{X}$ is a function defined by $F(x)=\hat{F}(x)\left(u_{i}\right) \forall u_{i} \in U$, where $B^{X}$ is the collection of all bipolar fuzzy sets. Then, $F(x)$ is called a bipolar fuzzy soft expert set.

Definition 3.11 [6]. A pair $(F, A)_{D}$ is called a fuzzy parameterized soft expert set over $U$, where $F$ is a mapping given by 
$F_{D}: A \longrightarrow P(U)$, while $D$ is the fuzzy subset of the set of parameters $E$ and $P(U)$ denotes the power set of $U$.

\section{Fuzzy Parameterized Bipolar Fuzzy Soft Ex- pert Sets}

We discussed the notion of FPBFSES and established relevant properties in this section.

Definition 4.1. Let $U=\left\{u_{1}, u_{2}, u_{3}, \ldots, u_{n}\right\}$ be set of universe and $E=\left\{e_{1}, e_{2}, e_{3}, \ldots, e_{m}\right\}$ be set of parameters, let $A=\left\{x_{1}, x_{2}, x_{3}, \ldots, x_{i}\right\}$ be a set of experts (agents), and let $O=\{1=$ agree, $0=$ disagree $\}$ be a set of opinions. Let $Z=$ $D \times A \times O$, where $D$ a fuzzy subset of $E$ then $Z=D \times A \times O$ is defined as

$$
Z=\{(d, x, o): d \in D, x \in A, o \in O\}
$$

For the sake of simplicity in this paper, it is supposed that the set of opinions only consists of two values, namely, agree and disagree. However, it is possible to include other options in the set of opinions, including more specific opinions.

Definition 4.2. Let $G$ be a mapping given by $G_{D}: X \rightarrow$ $B^{U}$, where $B^{U}$ denotes the set of all bipolar fuzzy set on $U$. Then the pair $(G, X)_{D}$ is said to be FPBFSES over $U$.

Example 4.3. Let $U=\left\{u_{1}, u_{2}, u_{3}\right\}$ be the set of three cars under consideration. Let $e=$ beautiful, $e=$ cheap and $e=\operatorname{good}$ conditionare the parameters for decision, $E=\left\{e_{1}, e_{2}, e_{3}\right\}$, $A=\left\{x_{1}, x_{2}\right\}$ be the set of experts and $D=\left\{0.9 / e_{1}, 0.6 / e_{2}\right.$, $\left.0.2 / e_{3}\right\}$. The following information was obtained after getting the opinion of two experts:

$$
(G, X)_{D}=\left\{\begin{array}{l}
\left(\left(\frac{0.9}{e_{1}}, x_{1}, 1\right)=\left\{\left(\frac{u_{1}}{(0.9,-0.2)}, \frac{u_{2}}{(0.6,-0.4)}, \frac{u_{3}}{(0.3-0.9)}\right)\right\}\right) \\
\left.\left(\frac{0.6}{e_{2}}, x_{1}, 1\right)=\left\{\left(\frac{u_{1}}{(0.7,-0.5)}, \frac{u_{2}}{(0.4,-0.4)}, \frac{u_{3}}{(0.1,-0.7)}\right)\right\}\right) \\
\left(\left(\frac{0.2}{e_{3}}, x_{1}, 1\right)=\left\{\left(\frac{u_{1}}{(1,-0.2)}, \frac{u_{2}}{(0.6,-0.4)}, \frac{u_{3}}{(0.3,-0.9)}\right)\right\}\right) \\
\left.\left(\frac{0.9}{e_{1}}, x_{2}, 1\right)=\left\{\left(\frac{u_{1}}{(0.8,-0.3)}, \frac{u_{2}}{(0.2,-0.7)}, \frac{u_{3}}{(0.8,-0.8)}\right)\right\}\right) \\
\left.\left.\left(\frac{0.6}{e_{2}}, x_{2}, 1\right)=\left\{\left(\frac{u_{1}}{(0.5,-0.5)}, \frac{u_{2}}{(0.8,-0.1)}, \frac{u_{3}}{(0.9,-0.2)}\right)\right\}\right)\right\} \\
\left.\left(\frac{0.2}{e_{3}}, x_{2}, 1\right)=\left\{\left(\frac{u_{1}}{(0.6,-0.6)}, \frac{u_{2}}{(0.2,-0.7)}, \frac{u_{3}}{(0.4,-0.1)}\right)\right\}\right) \\
\left.\left(\frac{0.9}{e_{1}}, x_{1}, 0\right)=\left\{\left(\frac{u_{1}}{(0.9,-0.2)}, \frac{u_{2}}{(0.8,-0.5)}, \frac{u_{3}}{(0.5,-0.7)}\right)\right\}\right) \\
\left(\left(\frac{0.6}{e_{2}}, x_{1}, 0\right)=\left\{\left(\frac{u_{1}}{(0.6,-0.4)}, \frac{u_{2}}{(0.5,-0.5)}, \frac{u_{3}}{(0.4,-0.7)}\right)\right\}\right) \\
\left(\left(\frac{0.9}{e_{1}}, x_{2}, 0\right)=\left\{\left(\frac{u_{1}}{(0.9,-0.5)}, \frac{u_{2}}{(0.3,-0.6)}, \frac{u_{3}}{(0.5,-0.7)}\right)\right\}\right) \\
\left(\left(\frac{0.2}{e_{3}}, x_{2}, 0\right)=\left\{\left(\frac{u_{1}}{(0.8,-0.5)}, \frac{u_{2}}{(0.7,-0.2)}, \frac{u_{3}}{(0.6,-0.5)}\right)\right\}\right)
\end{array}\right\} .
$$

Thus, $(G, X)_{D}$ is FPBFSES.

Definition 4.4. A $(G, X)_{D}$ is said to be FPBFSE subset of
$(H, Y)_{K}$, denoted by $(G, X)_{D} \sqsubseteq(H, Y)_{K}$ if

1) $X \subseteq Y$,

2) For all $\varepsilon \in X, G_{D}(\varepsilon)$ is bipolar fuzzy subset of $H_{K}(\varepsilon)$.

They are equal if $(G, X)_{D} \sqsubseteq(H, Y)_{K}$ and $(H, Y)_{K} \sqsubseteq$ $(G, X)_{D}$

Definition 4.5. An agree-FPBFSES denoted by $(G, X)_{D_{1}}$ is defined as

$$
(G, X)_{D_{1}}=\left\{G_{D}(\varepsilon): \varepsilon \in D \times A \times\{1\}\right\} .
$$

Definition 4.6. A disagree-FPBFSES denoted by $(G, X)_{D_{0}}$ is defined as

$$
(G, X)_{D_{0}}=\left\{G_{D}(\varepsilon): \varepsilon \in D \times A \times\{0\}\right\} .
$$

\section{Basic Operations on Fuzzy Parameterized Bipolar Fuzzy Soft Expert Sets}

Some basic operations such as complement, union, intersection, AND operator and OR operator of FPBFSES are discussed in this segment.

Definition 5.1. Let $(F, X)_{D}$ be a FPBFSES over $U$. Then, the complement of $(F, X)_{D}$, denoted by $(F, X)_{D}^{c}$, is defined as $(F, X)_{D}^{c}=\left(F^{c}, \sim X\right)_{D}$, where $F_{D}^{c}: \sim X \longrightarrow B^{U}$ is a mapping given by:

$$
F_{D}^{c}(\varepsilon)=c\left(F_{D}(\varepsilon)\right) \forall \varepsilon \in X,
$$

where $c$ is the bipolar fuzzy complement and $\sim X \subset D^{c} \times$ $A \times O$.

Example 5.2. Let $(F, X)_{D}$ be a FPBFSES as defined in Example 3.3. Then, using the complement of bipolar fuzzy set, we have

$$
(F, X)_{D}^{c}=\left\{\begin{array}{l}
\left(\left(\frac{0.1}{e_{1}}, x_{1}, 1\right)=\left\{\left(\frac{u_{1}}{(0.1,-0.8)}, \frac{u_{2}}{(0.4,-0.6)}, \frac{u_{3}}{(0.7,-0.1)}\right)\right\}\right) \\
\left(\left(\frac{0.4}{e_{2}}, x_{1}, 1\right)=\left\{\left(\frac{u_{1}}{(0.3,-0.5)}, \frac{u_{2}}{(0.6,-0.6)}, \frac{u_{3}}{(0.9,-0.3)}\right)\right\}\right) \\
\left(\left(\frac{0.8}{e_{3}}, x_{1}, 1\right)=\left\{\left(\frac{u_{1}}{(0,-0.8)}, \frac{u_{2}}{(0.4,-0.6)}, \frac{u_{3}}{(0.7,-0.1)}\right)\right\}\right) \\
\left.\left(\frac{0.1}{e_{1}}, x_{2}, 1\right)=\left\{\left(\frac{u_{1}}{(0.2,-0.7)}, \frac{u_{2}}{(0.8,-0.3)}, \frac{u_{3}}{(0.2,-0.2)}\right)\right\}\right) \\
\left.\left(\frac{0.4}{e_{2}}, x_{2}, 1\right)=\left\{\left(\frac{u_{1}}{(0.5,-0.5)}, \frac{u_{2}}{(0.2,-0.9)}, \frac{u_{3}}{(0.1,-0.8)}\right)\right\}\right) \\
\left.\left.\left(\frac{0.8}{e_{3}}, x_{2}, 1\right)=\left\{\left(\frac{u_{1}}{(0.4,-0.4)}, \frac{u_{2}}{(0.8,-0.3)}, \frac{u_{3}}{(0.6,-0.9)}\right)\right\}\right)\right\} \\
\left(\left(\frac{0.1}{e_{1}}, x_{1}, 0\right)=\left\{\left(\frac{u_{1}}{(0.1,-0.8)}, \frac{u_{2}}{(0.2,-0.5)}, \frac{u_{3}}{(0.5,-0.3)}\right)\right\}\right) \\
\left.\left(\frac{0.4}{e_{2}}, x_{1}, 0\right)=\left\{\left(\frac{u_{1}}{(0.4,-0.6)}, \frac{u_{2}}{(0.5,-0.5)}, \frac{u_{3}}{(0.6,-0.3)}\right)\right\}\right) \\
\left(\left(\frac{0.1}{e_{1}}, x_{2}, 0\right)=\left\{\left(\frac{u_{1}}{(0.1,-0.5)}, \frac{u_{2}}{(0.7,-0.4)}, \frac{u_{3}}{(0.5,-0.3)}\right)\right\}\right) \\
\left(\left(\frac{0.8}{e_{3}}, x_{2}, 0\right)=\left\{\left(\frac{u_{1}}{(0.2,-0.5)}, \frac{u_{2}}{(0.3,-0.8)}, \frac{u_{3}}{(0.4,-0.5)}\right)\right\}\right)
\end{array}\right\} .
$$


Proposition 5.3. Let $(F, X)_{D}$ be a FPBFSES then we have $\left((F, X)_{D}^{c}\right)^{c}=(F, X)_{D}$.

Proof: Let $(F, X)_{D}$ be a FPBFSES. Then by definition of complement we have a mapping $\left(F_{D}^{c}\right)^{c}: \sim(\sim X) \longrightarrow B^{U}$ defined by $\left(F_{D}^{c}\right)^{c}(\varepsilon)=c\left(F_{D}^{c}(\sim(\sim \varepsilon))\right)$ for all $\varepsilon \in \sim(\sim X)$ and $\sim(\sim X) \subseteq\left(\left(D^{c}\right)^{c} \times A \times O\right)$, since $\left(D^{c}\right)^{c}=D$, it's prove that $\sim(\sim X)=X$ and thus $\left((F, X)_{D}^{c}\right)^{c}=(F, X)_{D}$.

Definition 5.4. The union of two FPBFSESs $(G, X)_{D}$ and $(H, Y)_{K}$ over $U$, denoted by $(G, X)_{D} \cup(H, Y)_{K}$, is the FPBFSES $(I, Z)_{R}$ such that $Z=\{R \times A \times O\}$, where $R=D \cup K$ and $\forall \varepsilon \in Z$. Then $I_{R}(\varepsilon)$ can be defined as follows:

$$
I_{R}(\varepsilon)=G_{D}(\varepsilon) \bigcup H_{K}(\varepsilon),
$$

where $\bigcup$ is bipolar fuzzy soft union.

Example 5.5. Let $U=\left\{u_{1}, u_{2}, u_{3}\right\}$ be set of universe, $Z=\left\{e_{1}, e_{2}, e_{3}\right\}$ be set of decision parameter and $A=\left\{x_{1}\right.$, $\left.x_{2}\right\}$ be set of experts such that $X=\left\{e_{1}, e_{2}\right\}$ and $Y=\left\{e_{1}\right.$, $\left.e_{3}\right\}$. Suppose that $(G, X)_{D}$ and $(H, Y)_{K}$ are two FPBFSESs over a soft universe $U$ such that

$(G, X)_{D}=\left\{\begin{array}{l}\left(\left(\frac{0.8}{e_{1}}, x_{1}, 1\right)=\left\{\left(\frac{u_{1}}{(0.3,-0.4)}, \frac{u_{2}}{(0.6,-0.4)}, \frac{u_{3}}{(0.5,-0.6)}\right)\right\}\right) \\ \left.\left(\frac{0.8}{e_{1}}, x_{1}, 0\right)=\left\{\left(\frac{u_{1}}{(0.6,-0.6)}, \frac{u_{2}}{(0.7,-0.4)}, \frac{u_{3}}{(0.2,-0.9)}\right)\right\}\right) \\ \left.\left(\frac{0.6}{e_{2}}, x_{2}, 1\right)=\left\{\left(\frac{u_{1}}{(0.7,-0.5)}, \frac{u_{2}}{(0.8,-0.3)}, \frac{u_{3}}{(0.3,-0.7)}\right)\right\}\right) \\ \left(\left(\frac{0.6}{e_{2}}, x_{2}, 0\right)=\left\{\left(\frac{u_{1}}{(0.3,-0.7)}, \frac{u_{2}}{(0.2,-0.6)}, \frac{u_{3}}{(0.9,-0.4)}\right)\right\}\right)\end{array}\right\}$,

and

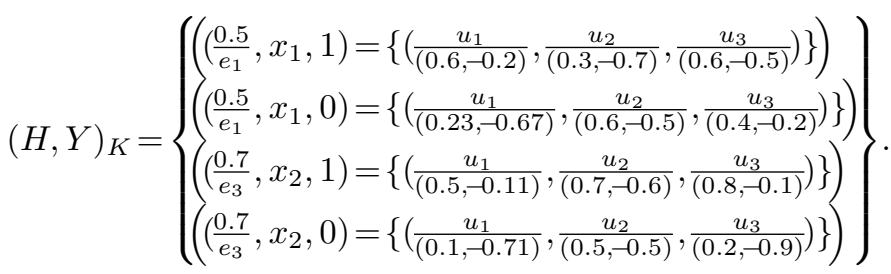

Using FPBFSES union, we have $(G, X)_{D} \uplus(H, Y)_{K}=(I, Z)_{R}$ where

$$
(I, Z)_{R}=\left\{\begin{array}{l}
\left(\left(\frac{0.5}{e_{1}}, x_{1}, 1\right)=\left\{\left(\frac{u_{1}}{(0.6,-0.4)}, \frac{u_{2}}{(0.6,-0.7)}, \frac{u_{3}}{(0.6,-0.6)}\right)\right\}\right) \\
\left(\left(\frac{0.5}{e_{1}}, x_{1}, 0\right)=\left\{\left(\frac{u_{1}}{(0.6,-0.67)}, \frac{u_{2}}{(0.7,-0.5)}, \frac{u_{3}}{(0.4,-0.9)}\right)\right\}\right) \\
\left.\left(\frac{0.6}{e_{2}}, x_{2}, 1\right)=\left\{\left(\frac{u_{1}}{(0.7,-0.5)}, \frac{u_{2}}{(0.8,-0.3)}, \frac{u_{3}}{(0.3,-0.7)}\right)\right\}\right) \\
\left.\left(\frac{0.6}{e_{2}}, x_{2}, 0\right)=\left\{\left(\frac{u_{1}}{(0.3,-0.7)}, \frac{u_{2}}{(0.2,-0.6)}, \frac{u_{3}}{(0.9,-0.4)}\right)\right\}\right) \\
\left(\left(\frac{0.7}{e_{3}}, x_{2}, 1\right)=\left\{\left(\frac{u_{1}}{(0.5,-0.11)}, \frac{u_{2}}{(0.7,-0.6)}, \frac{u_{3}}{(0.8,-0.1)}\right)\right\}\right) \\
\left(\left(\frac{0.7}{e_{3}}, x_{2}, 0\right)=\left\{\left(\frac{u_{1}}{(0.1,-0.71)}, \frac{u_{2}}{(0.5,-0.5)}, \frac{u_{3}}{(0.2,-0.9)}\right)\right\}\right)
\end{array}\right\} .
$$

Proposition 5.6 Let $(G, X)_{D},(H, Y)_{K}$ and $(I, Z)_{R}$ are FPBFSESs over a universe $U$. Then the following conditions hold:

1) $(G, X)_{D}$ ש $(H, Y)_{K}=(H, Y)_{K}$ ש $(G, X)_{D}$,

2) $\left((G, X)_{D} \mathbb{U}(H, Y)_{K}\right) \mathbb{U}(I, Z)_{R}=(G, X)_{D} \mathbb{U}\left((H, Y)_{K} \mathbb{U}\right.$ $\left.(I, Z)_{R}\right)$,

3) $(G, X)_{D} \cup(G, X)_{D}=(G, X)_{D}$.

Proof. Let $(G, X)_{D} \cup(H, Y)_{K}=(I, Z)_{R}$. Then by Definition 4.4, for all $\varepsilon \in Z$, we have $R=D \cup K$ and $I_{R}(\varepsilon)=$ $G_{D}(\varepsilon) \cup H_{K}(\varepsilon)$. As we know that the union of fuzzy sets and $\mathrm{BF}$ sets are commutative then $R=K \cup D$ and $I_{R}(\varepsilon)=$ $H_{K}(\varepsilon) \bigcup G_{D}(\varepsilon)$. Therefore $(I, Z)_{R}=(H, Y)_{K} \uplus(G, X)_{D}$. Hence $(G, X)_{D} \uplus(H, Y)_{K}=(H, Y)_{K} \uplus(G, X)_{D}$.

The proof of the remaining parts is straightforward and is therefore omitted.

Definition 5.7. The intersection of two FPBFSESs $(G, X)_{D}$ and $(H, Y)_{K}$ over $U$, denoted by $(G, X)_{D} \cap(H, Y)_{K}$, is the FPBFSES $(I, Z)_{R}$ such that $Z=\{R \times A \times O\}$, where $R=$ $D \cap K$ and $\forall \varepsilon \in Z$. Then, $I_{R}(\varepsilon)$ can be defined as

$$
I_{R}(\varepsilon)=G_{D}(\varepsilon) \bigcap H_{K}(\varepsilon),
$$

where $\bigcap$ is bipolar fuzzy soft intersection.

Example 5.8. Consider Example 5.5. Then, $(G, X)_{D}$ ก $(H, Y)_{K}=(I, Z)_{R}$, where

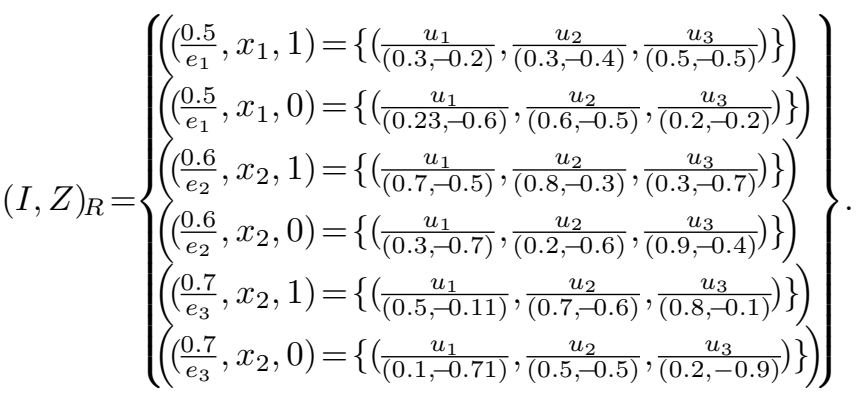

Proposition 5.9. Let $(G, X) D,(H, Y) K$, and $(I, Z) R$ be FPBFSESs over a universe $U$. Then, the following conditions hold:

$$
\begin{aligned}
& \text { 1) }(G, X)_{D} \cap(H, Y)_{K}=(H, Y)_{K} \text { ก }(G, X)_{D}, \\
& \text { 2) }\left((G, X)_{D} \uplus(H, Y)_{K}\right) \cap(I, Z)_{R}=(G, X)_{D} \cap\left((H, Y)_{K} \cap\right. \\
& \left.(I, Z)_{R}\right) \\
& \text { 3) }(G, X)_{D} \cap(G, X)_{D}=(G, X)_{D} .
\end{aligned}
$$

Proof. Its proof is straightforward by Definition 4.7 and is therefore omitted.

Proposition 5.10. Let $(G, X) D,(H, Y) K$, and $(I, Z) R$ be FPBFSESs over a universe $U$. Then, the following conditions hold:

1) $(G, X)_{D} \cap\left((H, Y)_{K} \uplus(I, Z)_{R}\right)=\left((G, X)_{D} \cap(H, Y)_{K}\right)$ ש $\left((G, X)_{D} \cap(I, Z)_{R}\right)$, 
2) $(G, X)_{D} \mathbb{U}\left((H, Y)_{K} \cap(I, Z)_{R}\right)=\left((G, X)_{D} \uplus(H, Y)_{K}\right)$ ก $\left((G, X)_{D} \uplus(I, Z)_{R}\right)$.

Proof. The proof is simple and is therefore omitted.

Proposition 5.11. Let $(G, X)_{D}$ and $(H, Y)_{K}$ are FPBFSESs over a universe $U$. Then the De Morgan's laws holds true:

1) $\left((G, X)_{D} \uplus(H, Y)_{K}\right)^{c}=(G, X)_{D}^{c} \cap(H, Y)_{K}^{c}$,

2) $\left((G, X)_{D} \cap(H, Y)_{K}\right)^{c}=(G, X)_{D}^{c}$ ש $(H, Y)_{K}^{c}$.

Proof. Let $(G, X)_{D}$ and $(H, Y)_{K}$ are FPBFSESs over a universe $U$, we have

$$
\begin{aligned}
(G, X)_{D}^{c} \cap(H, Y)_{K}^{c}= & G_{D}^{c}(\varepsilon) \bigcap H_{K}^{c}(\varepsilon) \\
& =c\left(G_{D}(\sim \varepsilon)\right) \bigcap c\left(H_{K}(\sim \varepsilon)\right) \\
& =c\left(G_{D}(\sim \varepsilon) \bigcap H_{K}(\sim \varepsilon)\right) \\
& =c\left(G_{D}(\varepsilon) \bigcup H_{K}(\varepsilon)\right) \\
& =\left((G, X)_{D} \uplus(H, Y)_{K}\right)^{c} .
\end{aligned}
$$

ii) The proof is simple and is therefore eliminated.

Definition 5.12. Let $(G, X)_{D}$ and $(H, Y)_{K}$ are FPBFSESs over a universe $U$. Then " $(G, X)_{D}$ AND $(H, Y)_{K}$ ", denoted by $(G, X)_{D} \hat{\wedge}(H, Y)_{K}=(I, X \times Y)_{R}$ is a FPBFSES such that $I_{R}(\alpha, \beta)=\left(G_{D}(\alpha) \cap H_{K}(\beta)\right)$ for all $(\alpha, \beta) \in X \times Y$ and $R=D \times K$.

Definition 5.13. Let $(G, X)_{D}$ and $(H, Y)_{K}$ are FPBFSESs over a universe $U$. Then " $(G, X)_{D}$ OR $(H, Y)_{K}$ ", denoted by $(G, X)_{D} \hat{V}(H, Y)_{K}=(I, X \times Y)_{R}$ is a FPBFSES such that $I_{R}(\alpha, \beta)=\left(G_{D}(\alpha) \cup H_{K}(\beta)\right)$ for all $(\alpha, \beta) \in X \times Y$ and $R=D \times K$.

Proposition 5.14. Let $(G, X)_{D}$ and $(H, Y)_{K}$ are FPBFSESs over a universe $U$. Then the De Morgan's laws holds true:

$$
\begin{aligned}
& \text { 1) }\left((G, X)_{D} \hat{\wedge}(H, Y)_{K}\right)^{c}=(G, X)_{D}^{c} \hat{\vee}(H, Y)_{K}^{c}, \\
& \text { 2) }\left((G, X)_{D} \hat{\vee}(H, Y)_{K}\right)^{c}=(G, X)_{D}^{c} \hat{\wedge}(H, Y)_{K}^{c} .
\end{aligned}
$$

Proof: 1$)$ Let $(G, X)_{D}$ and $(H, Y)_{K}$ are FPBFSESs over a universe $U$. Then by Definition 5.10, we have

$$
\begin{aligned}
\left((G, X)_{D} \hat{\wedge}(H, Y)_{K}\right)^{c} & =\left(\left(G_{D}(\alpha) \bigcap\left(H_{K}(\beta)\right)^{c}\right.\right. \\
& =G_{D}^{c}(\alpha) \bigcup H_{K}^{c}(\beta) \\
& =c\left(G_{D}(\sim \alpha)\right) \bigcup c\left(H_{K}(\sim \beta)\right) \\
& =(G, X)_{D}^{c} \hat{\vee}(H, Y)_{K}^{c} .
\end{aligned}
$$

ii) The proof is simple and is therefore eliminated.

\section{Application of FPBFSES in Decision Making}

To solve hypothetical decision-making problems, we established a generalized algorithm, which will be applied to a FPBFSES model in this section.

Suppose that $U=\{h 1, h 2, h 3\}$ is the set of three houses making the set of universe. Suppose that Mr. X wants to buy a house while considering four decision parameters $E=\{e 1$, $e 2, e 3, e 4\}$, where $e i(1,2,3,4)$ represents the aesthetics, build material, environment, and commute convenience, respectively. Mr. X, his wife, and son have their own opinions, which are represented by the set of experts $X=\{p, q, r\}$. After detailed observation, the family constructs the fuzzy sets

$$
D=\left\{\left(\frac{0.9}{e_{1}}, \frac{0.4}{e_{2}}, \frac{0.8}{e_{3}}\right)\right\},
$$

and this is subsequently used to form the following FPBFSES.

$$
(F, Z)_{D}=\left\{\begin{array}{l}
\left(\left(\frac{0.9}{e_{1}}, p, 1\right)=\left\{\left(\frac{u_{1}}{(0.5,-0.3)}, \frac{u_{2}}{(0.5,-0.4)}, \frac{u_{3}}{(0.7,-0.6)}\right)\right\}\right) \\
\left.\left(\frac{0.4}{e_{2}}, p, 1\right)=\left\{\left(\frac{u_{1}}{(0.6,-0.3)}, \frac{u_{2}}{(0.7,-0.5)}, \frac{u_{3}}{(0.2,-0.8)}\right)\right\}\right) \\
\left.\left(\frac{0.8}{e_{3}}, p, 1\right)=\left\{\left(\frac{u_{1}}{(0.5,-0.6)}, \frac{u_{2}}{(0.3,-0.5)}, \frac{u_{3}}{(0.6,-0.6)}\right)\right\}\right) \\
\left.\left(\frac{0.5}{e_{4}}, p, 1\right)=\left\{\left(\frac{u_{1}}{(0.9,-0.4)}, \frac{u_{2}}{(0.7,-0.7)}, \frac{u_{3}}{(0.5-0.3)}\right)\right\}\right) \\
\left(\left(\frac{0.9}{e_{1}}, q, 1\right)=\left\{\left(\frac{u_{1}}{(0.7,-0.8)}, \frac{u_{2}}{(0.2,-0.1)}, \frac{u_{3}}{(1,-0.4)}\right)\right\}\right) \\
\left.\left(\frac{0.4}{e_{2}}, q, 1\right)=\left\{\left(\frac{u_{1}}{(0.9,-0.6)}, \frac{u_{2}}{(0,-1)}, \frac{u_{3}}{(0.8,-0.5)}\right)\right\}\right) \\
\left(\left(\frac{0.8}{e_{3}}, q, 1\right)=\left\{\left(\frac{u_{1}}{(0.1,-0.6)}, \frac{u_{2}}{(0.6,-0.2)}, \frac{u_{3}}{(0.7,-0.5)}\right)\right\}\right) \\
\left(\left(\frac{0.5}{e_{4}}, q, 1\right)=\left\{\left(\frac{u_{1}}{(0.7,-0.5)}, \frac{u_{2}}{(0.8,-0.6)}, \frac{u_{3}}{(0.4,-0.5)}\right)\right\}\right) \\
\left.\left(\frac{0.9}{e_{1}}, r, 1\right)=\left\{\left(\frac{u_{1}}{(0.4,-0.8)}, \frac{u_{2}}{(0.8,-0.2)}, \frac{u_{3}}{(0.3,-0.5)}\right)\right\}\right) \\
\left.\left(\frac{0.5}{e_{2}}, r, 1\right)=\left\{\left(\frac{u_{1}}{(0.8,-0.78)}, \frac{u_{2}}{(0.7,-0.5)}, \frac{u_{3}}{(0.3,-0.66)}\right)\right\}\right) \\
\left(\left(\frac{0.9}{e_{1}}, p, 0\right)=\left\{\left(\frac{u_{1}}{(0.1,-0.1)}, \frac{u_{2}}{(0.7,-0.5)}, \frac{u_{3}}{(0.8,-0.58)}\right)\right\}\right) \\
\left(\left(\frac{0.4}{e_{2}}, p, 0\right)=\left\{\left(\frac{u_{1}}{(0.7,-0.4)}, \frac{u_{2}}{(0.6,-0.7)}, \frac{u_{3}}{(0.8,-0.3)}, \frac{u_{2}}{(0.11,-0.44)}, \frac{u_{3}}{(0.82,-0.45)}\right)\right\}\right) \\
\left.\left(\frac{0.8}{e_{3}}, p, 0\right)=\left\{\left(\frac{u_{1}}{(0.36,-0.7)}, \frac{u_{2}}{(0.8,-0.2)}, \frac{u_{3}}{(0.2,-0.7)}\right)\right\}\right) \\
\left(\left(\frac{0.9}{e_{1}}, q, 0\right)=\left\{\left(\frac{u_{1}}{(0.6,-0.9)}, \frac{u_{2}}{(0.2,-0.8)}, \frac{u_{3}}{(0.8,-0.1)}\right)\right\}\right) \\
\left.\left(\frac{0.4}{e_{2}}, q, 0\right)=\left\{\left(\frac{u_{1}}{(0.8,-0.8)}, \frac{u_{2}}{(0.9,-0.2)}, \frac{u_{3}}{(0.7,-0.5)}\right)\right\}\right) \\
\left(\left(\frac{0.5}{e_{4}}, q, 0\right)=\left\{\left(\frac{u_{1}}{(0.8,-0.3)}, \frac{u_{2}}{(0.8,-0.5)}, \frac{u_{3}}{(0.7,-0.1)}\right)\right\}\right) \\
\left(\left(\frac{0.9}{e_{1}}, r, 0\right)=\left\{\left(\frac{u_{1}}{(0.8,-0.4)}, \frac{u_{2}}{(0.8,-0.2)}, \frac{u_{3}}{(0.9,-0.5)}\right)\right\}\right) \\
\left.\left(\frac{0.8}{e_{3}}, r, 0\right)=\left\{\left(\frac{u_{1}}{(0.1,-0.7)}, \frac{u_{2}}{(0.8,-0.2)}, \frac{u_{3}}{(0.5,-0.8)}\right)\right\}\right) \\
\left(\left(\frac{0.5}{e_{4}}, r, 0\right)=\left\{\left(\frac{u_{1}}{(0.7,-0.4)}, \frac{u_{2}}{(0.9,-0.4)}, \frac{u_{3}}{(0.6,-0.5)}\right)\right\}\right)
\end{array}\right\},
$$

$A_{j}=\sum_{x \in X} \sum_{i=1}^{4} \mathrm{c}_{i j} \mu\left(e_{j}\right):$ Score $u_{1}=7.222$, score $u_{2}=6.42$, 
Table 1. Value of $c_{i}=f_{i}^{+}\left(u_{i}\right)-f_{i}^{-}\left(u_{i}\right)$ for all $u_{i} \in U$

\begin{tabular}{cccc}
\hline$U$ & $u_{1}$ & $u_{2}$ & $u_{3}$ \\
\hline$\left(0.9 / e_{1}, p, 1\right)$ & 0.8 & 0.9 & 1.3 \\
$\left(0.4 / e_{2}, p, 1\right)$ & 0.9 & 1.2 & 1 \\
$\left(0.8 / e_{3}, p, 1\right)$ & 1.1 & 0.8 & 1.2 \\
$\left(0.5 / e_{4}, p, 1\right)$ & 1.3 & 1.4 & 0.8 \\
$\left(0.9 / e_{1}, q, 1\right)$ & 1.3 & 0.3 & 1.4 \\
$\left(0.4 / e_{2}, q, 1\right)$ & 1.05 & 1 & 1.3 \\
$\left(0.8 / e_{3}, q, 1\right)$ & 0.7 & 0.8 & 1.2 \\
$\left(0.5 / e_{4}, q, 1\right)$ & 1.3 & 1 & 1.4 \\
$\left(0.9 / e_{1}, r, 1\right)$ & 1.2 & 1 & 0.8 \\
$\left(0.4 / e_{2}, r, 1\right)$ & 1.58 & 1.2 & 0.96 \\
$\left(0.5 / e_{4}, r, 1\right)$ & 0.2 & 1.2 & 1.38 \\
$\left(0.9 / e_{1}, p, 0\right)$ & 1.1 & 1.1 & 1.2 \\
$\left(0.4 / e_{2}, p, 0\right)$ & 1 & 0.55 & 1.27 \\
$\left(0.8 / e_{3}, p, 0\right)$ & 1.6 & 1 & 0.9 \\
$\left(0.9 / e_{1}, q, 0\right)$ & 1.5 & 1 & 0.9 \\
$\left(0.4 / e_{2}, q, 0\right)$ & 1.6 & 1.1 & 1.2 \\
\hline$\left(0.5 / e_{4}, q, 0\right)$ & 1.1 & 1.02 & 0.8 \\
$\left(0.9 / e_{1}, r, 0\right)$ & 1.2 & 1 & 1.4 \\
$\left(0.8 / e_{3}, r, 0\right)$ & 0.8 & 1 & 1.3 \\
$\left(0.5 / e_{4}, r, 0\right)$ & 1.1 & 1.3 & 1.1 \\
\hline
\end{tabular}

$$
\text { score } u_{3}=8.164
$$

$D_{j}=\sum_{x \in X} \sum_{i=1}^{4} c_{i j} \mu\left(e_{j}\right):$ Score $u_{1}=7.48$, score $u_{2}=6.21$, score $u_{3}=6.848$

Next, the generalized algorithm is applied to find the solution to a decision-making problem. The algorithm is used to select the best house among the three different houses. The generalized algorithm is defined as follows:

(1) Input the FPBFSES $(F, A)_{D}$.

(2) Compute $f_{p}^{+}\left(u_{i}\right)-f_{p}^{-}\left(u_{i}\right)$ for every $u_{i} \in U$, where $f_{p}^{+}\left(u_{i}\right)$ represent the positive response and $f_{p}^{-}\left(u_{i}\right)$ represent the negative response about each $u_{i} \in U$ (Table 1).

(3) Compute the largest numerical grade for agree and disagree FPBFSES.

(4) Find the score of every $u_{i} \in U$ by taking the sum of the products of the numerical grade of each element with member function of fuzzy set $D$ for agree and disagree FPBFSES.

(5) Find $r_{j}$ where $r_{j}=A_{j}-B_{j}$ for every $u_{i} \in U$.

(6) Find $s$, where $s=\max _{u_{i} \in U} r_{j}$. Then choose $u_{i}$ as the
Table 2. Numerical grade for agree-FPBFSES

\begin{tabular}{cccc}
\hline$U$ & $u_{1}$ & $u_{2}$ & $u_{3}$ \\
\hline$\left(0.9 / e_{1}, p, 1\right)$ & 0.8 & 0.9 & 1.3 \\
$\left(0.4 / e_{2}, p, 1\right)$ & 0.9 & 1.2 & 1 \\
$\left(0.8 / e_{3}, p, 1\right)$ & 1.1 & 0.8 & 1.2 \\
$\left(0.5 / e_{4}, p, 1\right)$ & 1.3 & 1.4 & 0.8 \\
$\left(0.9 / e_{1}, q, 1\right)$ & 1.3 & 0.3 & 1.4 \\
$\left(0.4 / e_{2}, q, 1\right)$ & 1.05 & 1 & 1.3 \\
$\left(0.8 / e_{3}, q, 1\right)$ & 0.7 & 0.8 & 1.2 \\
$\left(0.5 / e_{4}, q, 1\right)$ & 1.3 & 1 & 1.4 \\
$\left(0.9 / e_{1}, r, 1\right)$ & 1.2 & 1 & 0.8 \\
$\left(0.4 / e_{2}, r, 1\right)$ & 1.58 & 1.2 & 0.96 \\
$\left(0.5 / e_{4}, r, 1\right)$ & 0.2 & 1.2 & 1.38 \\
\hline
\end{tabular}

Table 3. Numerical grade for disagree-FPBFSES

\begin{tabular}{cccc}
\hline$U$ & $u_{1}$ & $u_{2}$ & $u_{3}$ \\
\hline$\left(0.9 / e_{1}, p, 0\right)$ & 1.1 & 1.1 & 1.2 \\
$\left(0.4 / e_{2}, p, 0\right)$ & 1 & 0.55 & 1.27 \\
$\left(0.8 / e_{3}, p, 0\right)$ & 1.6 & 1 & 0.9 \\
$\left(0.9 / e_{1}, q, 0\right)$ & 1.5 & 1 & 0.9 \\
$\left(0.4 / e_{2}, q, 0\right)$ & 1.6 & 1.1 & 1.2 \\
$\left(0.5 / e_{4}, q, 0\right)$ & 1.1 & 1.02 & 0.8 \\
$\left(0.9 / e_{1}, r, 0\right)$ & 1.2 & 1 & 1.4 \\
$\left(0.8 / e_{3}, r, 0\right)$ & 0.8 & 1 & 1.3 \\
$\left(0.5 / e_{4}, r, 0\right)$ & 1.1 & 1.3 & 1.1 \\
\hline
\end{tabular}

Table 4. The score $r_{j}=A_{j}-B_{j}$

\begin{tabular}{ccc}
\hline$A_{j}$ & $B_{j}$ & $r_{j}$ \\
\hline score $u_{1}=7.222$ & score $u_{1}=7.480$ & -0.258 \\
score $u_{2}=6.420$ & score $u_{2}=6.210$ & 0.210 \\
score $u_{3}=8.164$ & score $u_{3}=6.848$ & 1.316 \\
\hline
\end{tabular}

best solution to the problem.

The largest numerical grade for the element in agree and disagree PBFSES are given in Tables 2 and 3, respectively.

Let $A_{j}$ and $B_{j}$ denote the score for every numerical grade of the agree and disagree FPBFSES, respectively.

These values are shown in Table 4. From the computations in Tables 1-4, we obtained $s=\max _{u_{i} \in U} r_{j}=r_{3}$. Based on the results we have obtained using the algorithm, Mr. X should buy the house $h_{3}$. 


\section{Conclusion}

In this paper, the notion of FPBFSES was introduced. The basic operations on FPBFSES were described. We verified De Morgan's laws and other relevant laws by using the properties of these operations. We presented a decision-making generalized algorithm using this methodology and finally applied it to solve a decision-making problem.

\section{Conflict of Interest}

No potential conflict of interest relevant to this article was reported.

\section{References}

[1] D. Molodtsov, "Soft set theory: first results," Computers \& Mathematics with Applications, vol. 37, no. 4-5, pp. 19-31, 1999. https://doi.org/10.1016/S0898-1221(99)00056-5

[2] P. K. Maji, P. Biswas, and A. R. Roy, "A fuzzy soft sets," Journal of Fuzzy Mathematics, vol. 9, no. 3, pp. 589-602, 2001.

[3] S. Alkhazaleh, A. R. Salleh, and N. Hassan, "Fuzzy parameterized interval-valued fuzzy soft set," Applied Mathematical Sciences, vol. 5, no. 67, pp. 3335-3346, 2001.

[4] N. Cagman and S. Enginoglu, "FP-soft set theory and its applications," Annals of Fuzzy Mathematics and Informatics, vol. 2, no. 2, pp. 219-226, 2011.

[5] N. Cagman, F. Citak, and S. Enginoglu, "Fuzzy parameterized fuzzy soft set theory and its applications," Turkish Journal of Fuzzy System, vol. 1, no. 1, pp. 21-35, 2010.

[6] M. Bashir and A. R. Salleh, "Fuzzy parameterized soft expert set," Abstract and Applied Analysis, vol. 2012, article no. 258361, 2012. http://dx.doi.org/10.1155/2012/ 258361

[7] A. Hazaymeh, I. B. Abdullah, Z. Balkhi, and R. Ibrahim, "Fuzzy parameterized fuzzy soft expert set," Applied Mathematical Sciences, vol. 6, no. 112, pp. 5547-5564, 2012.

[8] G. Selvachandran and A. R. Salleh, "Fuzzy parameterized intuitionistic fuzzy soft expert set: theory and its application in decision making," International Journal of Soft Computing, vol. 11, no. 2, pp. 52-63, 2016.
[9] K. M. Lee, "Bipolar-valued fuzzy sets and their operations," in Proceedings of International Conference on Intelligent Technologies, Bangkok, Thailand, 2000, pp. 307-312.

[10] S. Abdullah, M. Aslam, and K. Ullah, "Bipolar fuzzy soft sets and its applications in decision making problem," Journal of Intelligent \& Fuzzy Systems, vol. 27, no. 2, pp. 729-742, 2014. http://dx.doi.org/10.3233/IFS131031

[11] S. Alkhazaleh and A. R. Salleh, "Soft expert sets," Advances in Decision Sciences, vol. 2011, article no. 757868 , 2011. http://dx.doi.org/10.1155/2011/757868

[12] Y. Al-Qudah and N. Hassan, "Bipolar fuzzy soft expert set and its application in decision making," International Journal of Applied Decision Sciences, vol. 10, no. 2, pp. 175-191, 2017.

[13] J. H. Park, Y. C. Kwun, and M. J. Son, "Multiperson multi-attribute decision making problems based on interval-valued intuitionistic fuzzy information," International Journal of Fuzzy Logic and Intelligent Systems, vol. 10, no. 4, pp. 287-295, 2010. http://doi.org/10.5391/IJFIS 2010.10.4.287

[14] J. H. Park, Y. C. Kwun, and M. J. Son, “A generalized intuitionistic fuzzy soft set theoretic approach to decision making problems," International Journal of Fuzzy Logic and Intelligent Systems, vol. 11, no. 2, pp. 71-76, 2011. https://doi.org/10.5391/IJFIS.2011.11.2.071

[15] I. Beg and T. Rashid, "Group decision making using intuitionistic hesitant fuzzy sets," International Journal of Fuzzy Logic and Intelligent Systems, vol. 14, no. 3, pp. 181-187, 2014. http://dx.doi.org/10.5391/IJFIS.2014.14.3. 181

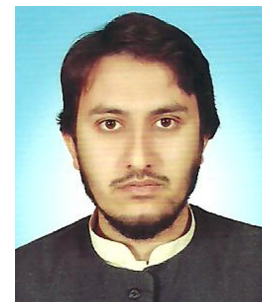

Muhammad Ibrar received his M.Sc. degree from department of Mathematics, Gomal University, M.Phil. degree from Quaid Azam University Islamabad, Pakistan in the field of fuzzy algebra. He is currently a Lecturer in department of Mathematics, University of Science and Technology Bannu, Pakistan. His research interest include biplar fuzzy sets theory, soft sets theory and decision making problems. 
E-mail: m.ibrar@ustb.edu.pk

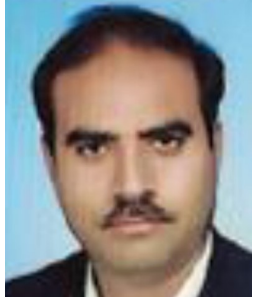

Asghar Khan received his M.Sc. degree from Department of Mathematics, University of Peshawar, M.Phil. and Ph.D. degrees from Quaid Azam University Islamabad, Pakistan in the field of fuzzy ordered semigroup. He is currently an Associate professor in Department of Mathematics, Abdul wali Khan University Mardan, Pakistan. His research interest include fuzzy algebras, fuzzy quantales, rough sets and soft sets with applications.

E-mail: azhar4set@yahoo.c

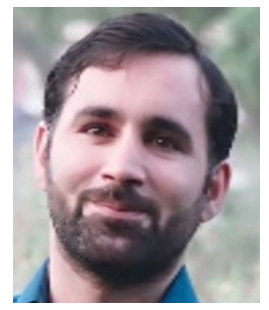

Sajjad Khan received the M.Sc. degree from department of Mathematics, University of Peshawar, M.Phil. and Ph.D. degrees from Hazara University, Pakistan in the field of Pythagorean fuzzy sets. He is currently Assistant professor in department of Mathematics, Hazara University, Pakistan. His research interest include fuzzy algebra, soft sets with application, multi attribute decision making problem.

E-mail: sajjadalimath@yahoo.com
Fatima Abbas received the his. and M.Phil. degrees from Department of Mathematics, Gomal University, Pakistan in the field of fuzzy sets. He is currently Assistant professor in Department of Mathematics, Gomal University, Pakistan. Her research interest include Fuzzy algebra, soft sets with application, multi attribute decision making problem. The photo is not included according to the author's request E-mail: Fatima.abass2@gmail.com 